\title{
The mechanism of COX-2 regulating HERG channel in gastric cancer cells
}

\author{
Shao XD, Guo XZ, Ren LN, Lin H \\ Department of Gastroenterology, General Hospital of Shenyang Military Area Command, Shenyang, China. \\ sxdsys608@sohu.com
}

\begin{abstract}
Objectives: To elucidate the signal transduction pathway, by which cyclooxygenase-2 (COX-2) regulates human erg-related gene (HERG) current in gastric cancer cells.

Methods: The HERG mRNA, protein and current in gastric cancer cells transfected with or without COX-2 antisense vector were measured by RT-PCR, Western blot and patch-clamp, respectively. Cyclic adenosine monophosphate (CAMP) concentration in gastric cancer cells transfected with or without COX-2 antisense vector was measured by ELISA.

Results: Transfection with COX-2 antisense vector did not alter the expression of HERG mRNA and protein, but it diminished the amplitude of HERG current in gastric cancer cells $(p<0.05)$. The cAMP concentration in gastric cancer cells transfected with COX-2 antisense vector was lower than that in parental gastric cancer cells ( $p$ $<0.05)$. COX-2 inhibitor and PGE2 had influence on the HERG current in gastric cancer cells. COX-2 inhibitor reduced the amplitude of HERG current in gastric cancer cells and PGE2 enhanced the amplitude. However, in gastric cancer cells transfected with HERG mutant deleting cAMP-binding domain, both COX-2 inhibitor and PGE2 did not show significant effects on HERG current. cAMP agonist enhanced the amplitude of HERG current and CAMP antagonist reduced the amplitude in gastric cancer cells. Both agonist and antagonist of cAMP had no significant effect on HERG current in gastric cancer cells transfected with HERG mutant deleting cAMP binding domain. PKA inhibitor did not influence the HERG current whether in parental gastric cancer cells or in gastric cancer cells transfected with HERG mutant.

Conclusions: COX-2 regulates HERG current through its catalytic product PGE2, which alters cAMP level in gastric cancer cells. cAMP interacts with HERG protein by binding with cAMP-binding domain of HERG protein and exerts impact on HERG current. PKA does not participate in this process (Tab. 1, Fig. 2, Ref. 18). Text in PDF www.elis.sk.

Key words: gastric cancer, COX-2, HERG, potassium channel, PGE2.
\end{abstract}

COX-2 plays an important role in carcinogenesis of several cancers, including colon cancer and gastric cancer. Some studies showed COX-2 was associated with the development of gastric cancer $(1,2)$ but its mechanism is still unclear. Our previous studies showed that COX-2 could affect the delayed rectifying potassium current in gastric cancer cells $(3,4)$ and the expression of asubunit of delayed rectifying potassium channel (HERG protein) was associated with biological behaviour of gastric cancer cells $(5,6)$. Based on the analysis of the structure of HERG protein and COX-2 related signal pathways, we designed the present study to explore the mechanism by which COX-2 regulates HERG current in gastric cancer cells.

Department of Gastroenterology, General Hospital of Shenyang Military Area Command, Shenyang, China

Address for correspondence: XD Shao, Department of Gastroenterology, General Hospital of Shenyang Military Area Command, No. 83, Wenhua Road, Shenhe District, Shenyang 110016, China. Fax: +86.24 .24685108$

Acknowledgements: This study was supported by grants from the Chinese National Foundation of Natural Science (No. 30400204).

\section{Materials and methods}

\section{Chemicals and reagents}

Celecoxib (Cearal; PGE2 (Sigma); 8-bromo-cAMP (Sigma); Rp-cAMP (Biolog Life Science); PKI (Sigma); LipofectmineTM 2000 (Invitrogen); T4 DNA ligase and restriction endonucleases (Takara Bio Co, Ltd.).

\section{Cell culture}

The human gastric cancer SGC7901 cell line, SGC7901 cell line transfected with COX-2 antisense vector (SGC7901-c, constructed by Han-Ping Wu) and human immortalized gastric epithelial GES cell line were cultured in RPMI 1640 medium containing $10 \%$ heat-inactivated fetal bovine serum and incubated at $37^{\circ} \mathrm{C}$ in a humidified atmosphere with $5 \% \mathrm{CO}_{2}$.

\section{RT-PCR}

Total RNA from subconfluent cultures of gastric cancer cells was isolated using Catrimox-14 RNA isolation kit (Takara Bio Co, Ltd.). RNA purity and integrity was checked by running an aliquot on a $1 \%$ agarose gel. $1-2 \mu \mathrm{g}$ were reverse transcribed in a $20 \mu \mathrm{l}$ reation by AMV Rtase (Takara Bio Co, Ltd.) 10U, $5 \times$ buffer 
$4 \mu \mathrm{l}$, dNTPs (ea.10 mM) $2 \mu$ l, RNAse Inhibitor $20 \mathrm{U}$ and Oligo (dT) $1850 \mathrm{pmol}$, for $1 \mathrm{~h}$ at $42{ }^{\circ} \mathrm{C} .5 \mu \mathrm{l}$ of cDNA were amplified by the polymerase chain reaction in a $50 \mu$ reaction containing

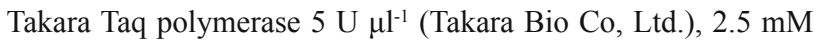
$\mathrm{MgCl}_{2}, 200 \mu \mathrm{M} \mathrm{d}(\mathrm{NTP}) \mathrm{s}, 1 \times \mathrm{PCR}$ buffer. The sequence of primers was as follows:

primer sense: 5'-TCCAGCGGCTGTACTCGGGC-3'

primer antisense: 5'-TGGACCAGAAGTGGTCGGAGAACTC-3'.

These primers comprise a sequence between nucleotide 2171 to nucleotide 2746 of the HERG sequence (accession number HU04270). PCR was performed as follows: 43 cycles $\left(94^{\circ} \mathrm{C}, 90 \mathrm{~s}\right.$; $65{ }^{\circ} \mathrm{C}, 180 \mathrm{~s} ; 72^{\circ} \mathrm{C}, 90 \mathrm{~s}$ ), followed by a final extension for 10 min at $72{ }^{\circ} \mathrm{C}$. $\beta$-actin served as an internal control. Products were run on a $1 \%$ agarose gel and bands were visualized by ethidium bromide staining on a UV transilluminator.

\section{Western blot}

Cell proteins were separated by SDS polyacrylamide gel electrophoresis and transferred to a nitrocellulose sheet. After transfer, the membranes were blocked for $4 \mathrm{~h}$ at room temperature with TBST containing $5 \%$ BSA and then incubated overnight at $4{ }^{\circ} \mathrm{C}$ with anti-HERG polyclonal antibody (Santa Cruz Bio) diluted 1:1000 in TBST. Membranes were then washed 3 times with TBST and incubated with anti-goat peroxidase-conjugate secondary antibodies for $1 \mathrm{~h}$ at room temperature. After 3 washes with TBST, the immunoreactivity was determined by a chemiluminescent reation. $\beta$-actin served as an internal control.

\section{Patch-clamp recordings}

Patch-clamp recordings were performed at room temperature with an amplifier Axonpatch 1-D (Axon Instruments). The whole cell configuration of the patch-clamp technique was employed, using pipettes whose resistance was in the range of 3-5M $\Omega$. Extracellular solutions were delivered through a remote-controlled linear positioner placed near the cell under study. The extracellular solution contained (mM): $\mathrm{NaCl} 95, \mathrm{KCl} 40, \mathrm{CaCl}_{2} 2$, Hepes 10 , glucose 5, $\mathrm{pH}$ 7.4. The standard pipette solution contained (mM): $\mathrm{KCl} 130, \mathrm{NaCl} 10, \mathrm{MgCl}_{2} 2, \mathrm{CaCl}_{2}$ 2, EGTA 10, Hepes 10, pH 7.4. Gigaseal resistance was in the range of 3-20 G $\Omega$. Whole cell currents were filtered at $5 \mathrm{kHz}$. Input resistance of the cells was in the range of $2-6 \mathrm{G} \Omega$.

\section{Measurement of cAMP concentration}

cAMP concentration in gastric cancer cells transfected with or without COX-2 antisense vector was measured by ELISA according to the instructions of the kit (B\&D, No DE0450). Total Activity (TA), Non-Specific Binding (NSB), Maximum Binding (B0), and Substrate Blank wells were run as a means of quality control for each assay.

Briefly, the culture media was removed by aspirating or decanting. The cells were lysed by adding $0.1 \mathrm{M} \mathrm{HCl}$. After incubation for 10 minutes, the cells were centrifuged at $600 \mathrm{x}$ g and the supernate was collected. Fifty $\mu$ l of cell supernates were added into $200 \mu$ l Assay Buffer ED2. One hundred and fifty $\mu$ l of Assay
Buffer were added into the NSB wells. One hundred $\mu$ l of Assay Buffer were added into B0 wells and $100 \mu 1$ of Standard or sample were added into the remaining cells. Fifty $\mu 1$ of cAMP conjugate were added into each well (excluding the TA and Substrate Blank wells). Fifty $\mu \mathrm{l}$ of cAMP Antibody Solution were added into each well (excluding the NSB, TA and Substrate Blank wells), followed by incubation for 2 hours at room temperature on a horizontal orbital microplate shaker set at $500 \mathrm{rpm}$. Each well was aspirated and washed, repeating the process twice for a total of three washes. Five $\mu$ l of cAMP conjugate into the TA wells. Two hundred $\mu$ l of pNPP substrate were added into all wells, followed by incubation for 1 hour at room temperature on the benchtop. Fifty $\mu$ of Stop Solution were added into each well. The optical density of each well was immediately determined using a microplate reader set to $405 \mathrm{~nm}$ with wavelength correction set between $570 \mathrm{~nm}$ and $590 \mathrm{~nm}$. The concentration of cAMP corresponding to the mean absorbance was calculated from the standard curve, which was generated according to the instructions of the kit.

\section{Construction of HERG mutant without cAMP-binding domain} Primers:

MF: 5'-TACTGTGACCTACACAAGATCC-3'

MR: 5'-ATCTTGTGTAGGTCACAGTACCTTGCATACAGGTTCAGAGGCTC-3'

\section{Sp6: 5'-CATACGATTTAGGTGACACTATAG-3'}

F1: 5'-GAGTTCATCCGCTTCCACCA-3'

The template was pcDNA3.0-HERG provided by Prof. Gail Robertson from Wisconsin-Madison University. Briefly, the product I and II of PCR were obtained using primers F1/MR and SP6/ $\mathrm{MF}$, respectively. Then the product I and II were mixed as template and product III was produced using primers F1/SP6. Product III was digested by XhoI restriction enzyme and purified as insert DNA. pcDNA3.0-HERG was digested by XhoI and the purified $7.0 \mathrm{~kb}$ segment was referred as vector DNA. Insert DNA and vector DNA were ligated using T4 DNA ligase and the recombinant was transformed into competent cells. Ampicillin-resistant colonies were chosen, identified by PCR using primers F1/M, and further confirmed by DNA sequencing.

\section{Transfection}

SGC7901 cells were planted into six-well plates and cultured in drug-free medium. At 90-95\% confluence, cells were washed twice with PBS and grown in 2ml DMEM without antibiotics. Using Lipofectamine 2000 reagent, two $\mu \mathrm{g}$ of plasmids were transfected into SGC7901cells according to the manufacturer's instructions. Forty-eight hours later, cells were placed in growth medium containing G418 (GIBCO) for clone selection. The positive clones were named as SGC7901 $\Delta$ cell line.

\section{The effects of different agents on HERG current in SGC7901 and SGC79014 cell line}

The SGC7901 and SGC7901 $\Delta$ cells were treated with Celecoxib $(320 \mu \mathrm{M})$, PGE2 $(100 \mu \mathrm{M}), 8$-bromo-cAMP $(500 \mu \mathrm{M})$ and Rp-cAMP $(100 \mu \mathrm{M})$ for 5 minutes, respectively. Then the HERG currents in the treated and control gastric cancer cells were mea- 
sured using patch clamp. The SGC7901 and SGC7901 $\Delta$ cells pretreated with PKI $(1 \mu \mathrm{M})$ for 5 minutes were treated with or without PGE2 $(100 \mu \mathrm{M})$ and the HERG currents in these cells were recorded.

\section{Statistical analysis}

Results are expressed as mean \pm standard deviation (SD). Statistical analyses were performed with SPSS 11.0 statistical software. Independent-Samples $t$-test was adopted. Significance was defined as $\mathrm{p}<0.05$.

\section{Results}

The effects of COX-2 on HERG $m R N A$, protein and current in gastric cancer cells

As shown in Figures 1 and 2, there was no difference in the expression of HERG mRNA and protein between SGC7901 and SGC7901-c cells, indicating that COX-2 does not influence the transcription and translation of HERG gene in gastric cancer cells. But the amplitude of HERG current in SGC7901-c $(63.2133 \pm 1.85648 \mathrm{pA})$ was lower than that in SGC7901 (82.6833 $\pm 1.58863 \mathrm{pA})(\mathrm{p}<0.05, \mathrm{n}=3)$.

The effect of $\mathrm{COX}-2$ on $\mathrm{CAMP}$ concentration in gastric cancer cells

The cAMP concetration in SGC7901-c cells (1.4533 \pm $0.41986 \mathrm{pM}$ ) was significantly lower than that in SGC7901 cells $(3.0167 \pm 0.78002 \mathrm{pM})(\mathrm{p}<0.05, \mathrm{n}=3)$.

\section{The construction of mutant HERG- $\triangle N B D$}

Electrophoresis showed that PCR product I, II and III presented with anticipated molecular weight. The mutant with proper insert was verified by PCR using primers F1/MR and confirmed by DNA sequencing. The deleted DNA sequence was 5'-CCT GGC AAG TCG AAC GGG GAT GTG CGG GCC CTC ACC-3', whose translation product would be PGKSNGDVRALT, which is the cAMP binding domain. The mutant was named as HERG- $\triangle$ NBD.

The effects of COX-2 inhibitor and signal molecules on HERG current in SGC7901 and SGC7901-A

As shown in Table 1, COX-2 inhibitor reduced the amplitude of HERG current and PGE2 enhanced the amplitude in SGC7901 cells. However, in SGC7901- $\Delta$ cells both, COX-2 inhibitor and PGE2 did not show significant effects on HERG current. cAMP agonist enhanced the amplitude of HERG current and cAMP antagonist reduced the amplitude in SGC7901 cells. Both agonist and

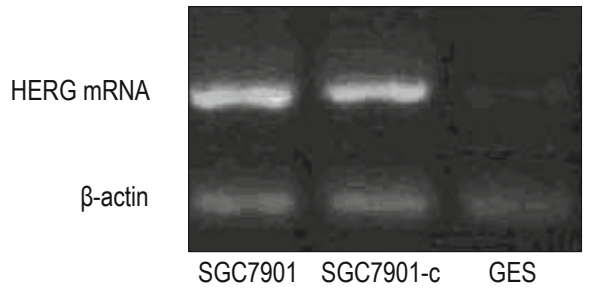

Fig. 1. The effect of COX-2 on HERG mRNA in gastric cancer cells.

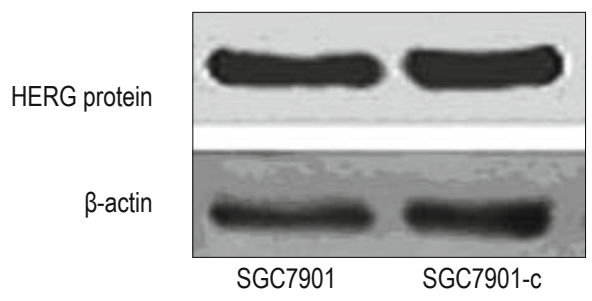

Fig. 2. The effect of COX-2 on HERG protein in gastric cancer cells.

antagonist of cAMP had no significant effect on HERG current in SGC7901- $\Delta$ cells. PKA inhibitor did not influence the HERG current whether in SGC7901 cells or in SGC7901- $\Delta$ cells.

\section{Discussion}

Our results showed that COX-2 did not affect the expression of HERG protein in gastric cancer cells but COX-2 did influence the HERG current in gastric cancer cells, indicating the regulation takes place after translation process. ELISA assay showed COX-2 increased the CAMP concentration in gastric cancer cells. Wu et al reported that COX-2 could increase the activity of PKA in gastric cancer cells, but the total amount of PKA was not changed (3). By deleting the cAMP binding domain in HERG protein and detecting the effects of different signal molecules on HERG current, we found that COX-2 could influence HERG current through cAMP without PKA involving.

COX enzymes catalyze the synthesis of prostaglandins (PGs) from arachidonic acid. The two isoforms of COX, COX-1 and COX-2, differ in many respects. COX-1 is expressed constitutively in most tissues, but COX-2 is not detected in most normal tissues. COX-2 is induced by mitogenic and inflammatory stimuli. Epidemiological studies showed that the use of non-steroidal anti-inflammatory drugs (NSAIDs), which inhibits the activity of COX-2, is associated with a reduced risk of several malignancies $(7,8)$. Consistent with this, tumor formation and growth are inhib-

Tab. 1. The effects of different agents on HERG current (pA) in SGC7901 and SGC7901 $\Delta$ cell line.

\begin{tabular}{|c|c|c|c|c|c|}
\hline \multirow[b]{2}{*}{ Cells } & \multicolumn{5}{|c|}{ Agents } \\
\hline & Celecoxib & PGE2 & 8-bromo-cAMP & Rp-cAMP & PKI+PGE2 \\
\hline$\overline{\text { SGC7901 }(n=3)}$ & $\begin{array}{l}\text { Treated: } 66.1333 \pm 3.04746^{*} \\
\text { Control: } 82.6833 \pm 1.58863\end{array}$ & $96.8667 \pm 1.49004^{*}$ & $91.8000 \pm 1.65375 *$ & $73.9133 \pm 1.47974^{*}$ & $95.8400 \pm 2.03266^{*}$ \\
\hline$\overline{\mathrm{SGC}} 7901 \Delta(\mathrm{n}=3)$ & $\begin{array}{l}\text { Treated: } 79.6267 \pm 0.96821 \\
\text { Control: } 80.8600 \pm 0.64490\end{array}$ & $79.8967 \pm 0.56889$ & $80.3600 \pm 0.56824$ & $81.1833 \pm 1.44140$ & $80.2433 \pm 0.34487$ \\
\hline
\end{tabular}


487-491

ited in animals that are either engineered to be COX-2 deficient or treated with a selective COX-2 inhibitor.

There are multiple mechanisms through which COX-2 may play a role in carcinogenesis. A number of these mechanisms are likely to result from COX-2-induced increase in PG synthesis. Increased PG synthesis has both, growth-promoting and positive feedback effects in cancer cells. Some studies provided evidence that increased expression of COX-2/PGE2 contributes to cancer development and progression via activation of the IL- 6 signaling pathway. COX-2 over-expression has been shown to up-regulate Bcl-2 expression with an associated decrease in apoptosis. In addition, COX-2 was found to be induced by TNF- $\alpha$, which underlines the inducibility of $\mathrm{COX}-2$ in response to pro-inflammatory stimulus. Other effects of COX-2 overexpression that may contribute to the malignant phenotype include decreased E-cadherin expression with consequent loss of cell-to-cell adhesion, matrixmetalloproteinase overexpression with an associated increase in invasiveness, and modulated production of angiogenic factors by cancer cells. Moreover, the COX-2-dependent effect on VEGF up-regulation was found to be inhibited by treatment with COX-2 specific inhibitor and this inhibitory effect was reversed by PGE2 treatment. Since VEGF plays an important role in angiogenesis, its up-regulation by COX-2 expression and inhibition by COX-2 specific inhibitor suggested a positive role of COX-2 in angiogenesis, an important event in cancer metastasis. Some studies suggested that overexpression of COX-2 in cancer cells places an additional burden on antioxidative defenses of the cell, which might contribute to DNA oxidation and the induction of mutation. Further, studies have also shown that overexpression of COX-2 in cancer cells inhibited immune surveillance and increased metastatic potential.

Our previous studies revealed that COX-2 is overexpressed in most gastric cancer tissues and associated with differentiation of gastric cancer. The overexpression of COX-2 was also found in gastric cancer cells accompanied with high PGs production (1). Inhibiting COX-2 could inhibit proliferation and increase apoptosis of gastric cancer cells (9). Our results also demonstrated that COX-2 was associated with delayed rectifying potassium current in gastric cancer cells and inhibiting expression or activity of COX-2 could diminish the delayed rectifying potassium current in gastric cancer cells $(3,4)$. Until now, there is no published report about the relationship between COX-2 and delayed rectifying potassium current in gastric cancer cells.

Most reports about COX-2 and delayed rectifying potassium channel are confined in the field of regulation of vascular tension and neuron. Some studies indicated that catalyzing products of COX-2 may regulate delayed rectifying potassium channel through cAMP. Several reports showed that PGs could activate delayed rectifying potassium channel. For example, in smooth muscle of the guinea-pig coronary artery the slow hyperpolarization is possibly produced by endothelial prostanoids through activation of delayed rectifying potassium channel, which could be blocked by COX2 inhibitor (10). A study on presynaptic modulation showed that COX-2 inhibitor could block delayed rectifying potassium channel and exogenous PGE2 could activate the channel implying that PGs positively modulate delayed rectifying potassium currents (11).
However, other studies showed that PGs inhibited delayed rectifying potassium channel. For example, in sensory neuron delayed rectifying potassium current was inhibited by PGE2 and regulated by PKA (12). PGs inhibited delayed rectifying potassium channel through PKA in megakaryocyte (13). These results revealed the different effects of PGs on delayed rectifying potassium channel in specific cells and different physiological processes.

The HERG protein contains a putative cyclic nucleotide binding domain (CNBD) in its cytoplasmic carboxyl terminus and four potential PKA phosphorylation sites, which means elevation of cAMP can modulate HERG channel by direct binding or indirect regulation through PKA. Over-expression of COX-2 can lead to cAMP elevations in gastric cancer cells. So we speculate that CNBD domain may play a role in the regulation of HERG current by COX-2 in gastric cancer cells. A previous study showed PKA can influence the HERG current (14). However, the existence of CNBD domain in the carboxyl terminus of HERG protein indicates that cAMP may directly regulate HERG channel independent of PKA. Cui et al measured the HERG current in CHO cells expressing cloned HERG and found decrease in HERG amplitude after introduction of cAMP analog. PKI, the specific PKA inhibitor, prevented the CAMP-mediated current reduction. After the four phosphorylation sites for PKA were mutagenized, cAMP can still affect the HERG current mediated by the non-PKA phosphorylatable mutants, indicating that cAMP may regulate the HERG current by dual pathways (15). Previous studies identified six invariant key amino acid residues for cyclic nucleotide binding. Three of these are glycine residues that are essential for maintenance of the $\beta$-barrel structure that is required to form a pocket for cyclic nucleotide binding. A glutamic acid residue forms a hydrogen bond with the ribose 2'-OH of cAMP and an arginine residue interacts with the phosphate of cAMP to form a salt bridge. Mutagenesis of any of these invariant amino acid residues impairs or eliminates cAMP binding. Mutant HERG proteins do not have a dominant-negative effect on wild-type current but do alter voltage-dependent gating. Compared with wild-type HERG homotetramer, co-expression of wild-type with mutant HERG did not significantly alter current amplitude. The mechanism by which CNBD affects HERG current is still unclear. One possibility is that CNBD binding with cAMP leads to the conformational change of HERG protein and then activates the potassium channel. Another possibility is that CNBD interacts with small proteins in cell membrane such as minK and KCNE2, which in turn affect potassium channel (16). Intracellular elevation of cAMP can regulate HERG current by directly binding to CNBD or indirectly via PKA pathway. Several studies indicated that various stimuli can affect HERG current by increasing intracellular cAMP (17). A study showed that HERG mutant deleting CNBD domain but preserving carboxyl terminus still can produce HERG current.(18) Based on our results and literature, we concluded that in gastric cancer cells COX-2 regulates HERG current through its catalytic product PGE2, which binds with its receptor on the gastric cancer cells and alters cAMP level in gastric cancer cells. cAMP interacts with HERG protein by binding with cAMP-binding domain of HERG protein and exerts an impact on HERG current. PKA does not participate in this process. 


\section{References}

1. Li L, Wu KC, Nie YZ, Wu HP, Wang CM, Fan DM. The expression and distribution of COX in human gastric cancer cells. Zhonghua Xiaohua Zazhi 2000; 20: 374-377.

2. Uefuji K, Ichikura T, Mochizuki H. Cyclooxygenase-2 expression is related to prostaglandin biosynthesis and angiogenesis in human gastric cancer. Clin Cancer Res 2000; 6: 135-138.

3. Wu HP, Wu KC, Han Y, Shi YQ, Yao LP, Wang J. The regulation of delayed rectifying potassium channel by COX-2 in gastric cancer cells. Zhonghua Zhongliu Zazhi 2002; 24: 440-443.

4. Han Y, Lang B, Wu HP, Zhou W, Gao Z, Zhou SS. The characteristics of potassium channel in human gastric cancer cell SGC7901 lines. Disi Junyi Daxue Xuebao 2002; 23: 608-610.

5. Shao XD, Wu KC, Hao ZM, Hong L, Zhang J, Fan DM. The potent inhibitory effects of cisapride, a specific blocker for human ether-a-gogo-related gene (HERG) channel, on gastric cancer cells. Cancer Biol Ther 2005; 4: 295-301.

6. Shao XD, Wu KC, Guo XZ, Xie MJ, Zhang J, Fan DM. Expression and significance of HERG protein in gastric cancer. Cancer Biol Ther 2008; 7: 45-50.

7. Neufang G, Furstenberger G, Heidt M, Marks F, Muller-Decker K. Abnormal differentiation of epidermis in transgenic mice constitutively expressing cyclooxygenase-2 in skin. PNAS 2001; 98: 7629-7634.

8. Zhu FS, Chen XM, Wang YJ. The effect of combination of specific COX inhibitor and anti-cancer drugs on the proliferation of gastric cancer cells. Zhonghua Zhongliu Zazhi 2007; 29: 186-188.

9. Xu MH, Wu KC, Wu HP, Yao LP, Fan DM. The inhibitory effect of NSAIDs on the proliferation of gastric cancer cells. Zhonghua Xiaohua Zazhi 2002; 22: 99-202.
10. Nishiyama M, Hashitani H, Fukuta H, Yamamoto Y, Suzuki H. Potassium channels activated in the endothelium-dependent hyperpolarization in guinea-pig coronary artery. J Physiol 1998; 510: 455-465.

11. Zoltay G, Cooper JR. Presynaptic modulation by eicosanoids in cortical synaptosomes. Neurochem Res 1994; 19: 1211-1215.

12. Evans AR, Vasko MR, Nicol GD. The cAMP transduction cascade mediates the PGE2-induced inhibition of potassium currents in rat sensory neurons. J Phsiol 19995; 16: 163-178.

13. Kapural L, Fein A. Suppression of the voltage-gated K+ current of human megakaryocytes by thrombin and prostacyclin. Biochim Biophys Acta 1997; 1355: 331-342.

14. Thomas D, Zhang W, Karle CA, Kathofer S, Schols W, Kubler W. Deletion of protein kinase A phosphorylation sites in the HERG potassium channel inhibits activation shift by protein kinase A. J Biol Chem 1999; 274: 27457-27462.

15. Cui J, Melman Y, Palma E, Fishman GI, McDonald TV. Cyclic AMP regulates the HERG K channel by dual pathways. Current Biology 2000; 10: 671-674.

16. Cui J, Kagan A, Qin D, Mathew J, Melman YF, McDonald TV. Analysis of the cyclic nucleotide binding domain of the HERG potassium channel and inerractions with KCNE2. J Biol Chem 2001; 276: 17244-17251.

17. Kagan A, Melman YF, Krumerman A. 14-3-3 amplifies and prolongs adrenergic stimulation of HERG potassium channel activity. EMBO J 2002; 21: 1889-1898.

18. Kupershmidt S, Snyders DJ, Raes A. A potassium channel splice variant common in human heart lacks a c-terminal domain required for expression of rapidly activating delayed rectifier current. J Biol Chem 1998; 273: 27231-27235.

Received June 16, 2013. Accepted April 12, 2014. 